\title{
Lipid Concentration Effect on Blood Flow Through an Inclined Arterial Channel with Magnetic Field
}

\author{
Kubugha Wilcox Bunonyo ${ }^{1}$, Emeka Amos ${ }^{2}$ \\ ${ }^{1}$ Department of Mathematics and Statistics, Federal University Otuoke, Yenagoa, Nigeria \\ ${ }^{2}$ Department of Mathematics, Rivers State University, Port Harcourt, Nigeria
}

Email address:

wilcoxbk@fuotuke.edu.ng (K. W. Bunonyo)

\section{To cite this article:}

Kubugha Wilcox Bunonyo, Emeka Amos. Lipid Concentration Effect on Blood Flow Through an Inclined Arterial Channel with Magnetic Field. Mathematical Modelling and Applications. Vol. 5, No. 3, 2020, pp. 129-137. doi: 10.11648/j.mma.20200503.11

Received: May 8, 2020; Accepted: May 22, 2020; Published: June 4, 2020

\begin{abstract}
The purpose of this research is to formulate mathematical models to investigate lipid concentration effect on blood flow though an inclined arterial channel with magnetic field. The formulated coupled partial differential equations were made dimensionless and reduced to ordinary differential equation using a perturbation technique, the nonlinear ordinary differential equations were solved analytically for the blood velocity and lipid concentration profiles respectively with some resultant pertinent parameters. Numerical simulations were carried out using Mathematica codes developed by the authors for the flow profiles by carefully varying the pertinent parameters to study the effect of each of the parameters on velocity and concentration profiles respectively. It is notice that the solutal Grashoff number, Darcy number, angle of inclination, time and the treatment parameters respectively causes the velocity profiles to increase, while the parameters such as the onset, length of stenosis, Schmidt number, magnetic field intensity, and pulse rate respectively decelerate the velocity profile. Secondly, the parameters such as length of stenosis, the pulse rate, Schmidt number and the treatment parameters decelerate the concentration profile while the onset parameter increases the concentration profile and the results were presented graphically. We can conclude that lipid concentration and some of the resulted pertinent parameters either increases or decreases the flow profiles and of great importance in studying blood velocity in arterial channel.
\end{abstract}

Keywords: Lipid, Blood, Flow, Artery, Magnetic Field, Concentration

\section{Introduction}

Blood flow refers to the movement of blood through a vessel, tissue, or organ, and is usually expressed in terms of volume of blood per unit of time. It is initiated by the contraction of the ventricles of the heart. Ventricular contraction ejects blood into the major arteries, resulting in flow from regions of higher pressure to regions of lower pressure, as blood encounters smaller arteries and arterioles, then capillaries, then the venules and veins of the venous system Bunonyo et al [1].

Atherosclerosis is the deposition or accumulation of cholesterol in the arterial wall and this can cause local narrowing in the lumen of the arterial segment commonly referred to as medically as stenosis. One of the serious consequences, when an obstruction is developed in an artery, is the increased resistance and the associated reduction of the blood flow which can lead to arterial diseases such as stroke, heart attack and serious circulating disorders Those diseases have identified as major deaths worldwide Shanthiet al. [2]. Since the normal blood flow is disturbed as a result of formation of lumps in the lumen of the arteries, the heat transfers between the living tissues particularly in the peripheral vessels where the temperature is generally closely related with blood flow rate will also be disturbed. Several studies on blood flow and heat transfer through stenosed arteries have been done theoretically and experimentally by several researchers [3-11]. Most of these studies considered only the magnetic field effect with no-slip boundary conditions. However, a number of studies of suspensions in general and blood flow in particular have both experimentally by Misra and Shit [12], Ponalgusamy [13]), and theoretically Verma et al. [14], Guar and Gupta [15] suggested the presence of slip at the flow boundaries. In a recent development, Srikanth, etal., [16] investigated blood flow through an overlapping clogged tapered artery in the 
presence of catheter. They considered velocity slip at the arterial wall since cholesterol deposition is resulting in the stenosis formation. They solved analytically the equation governing the fluid flow under the assumption of mild stenosis. Their results were presented graphically and from the graphs, it was observed that the slip velocity and divergence tapered artery facilitate the fluid flow. The effect of slip velocity on blood flow through an arterial tube in the presence of multiple stenosis was studied by Arun [17]. He considered the effects of length of stenosis and shape parameter on resistance to flow and shear stress. He observed from the graphs that the pertinent parameters have small variations for different values of stenosis shape parameter. An approximate perturbation scheme has been adopted by Geeta and Siddique [18] to solve the equations governing the unsteady blood flow through constrictedartery in the presence of velocity slip. They characterized the rheology of the blood flow by Bingham plastic fluids model. They considered the important flow parameters such as velocity, flow rate and shear stress and represented the results graphically. They concluded that, since high blood viscosity is very dangerous for the cardiovascular disorders, slip velocity at the stenotic wall may be used as the major tool in reducing the blood viscosity. Hematocrit effect on the axisymmetric blood flow through stenosed arteries has been investigated by Sanjeev and Chandrasekhar [19]. The mathematical model of blood flow through a tapered artery with mild stenosis and hematocrit were studied by Verma and Parihar [20]. Some of the above mentioned researchers only considered variable blood viscosity in their studies include Shit and Screenparna [21], Singh and Rathee [22]; Chitra and Karthikeyan [22]; Jagdish and Rajbala [24]. Bunonyo and Amos [25] investigated treatment and radiation effect on an atherosclerotic blood flow in an arterial channel with energy equation and solving the simulating the function by varying some pertinent variables and studying their impacts on the temperature profile.

This research aims at investigating the lipid concentration effect on blood flow through an inclined irregular arterial channel with magnetic field which the above mentioned researchers didn't consider. We will be looking at the treatment parameter, effect of the length of stenosis, height of stenosis, onset of stenosisin our investigation, and to find out how they enhance blood flowin different treatment regime.

\section{Mathematical Formulation}

We formulate mathematical models to investigate the lipid concentration effect on blood flow through an inclined irregular channel with magnetic field by consider the following assumption: it is assumed that blood is an incompressible, viscous, Newtonian and homogenous fluid which flows through an inclined irregular channel with normal radius, $R_{0}$. In addition, magnetic field is perpendicularly applied to the direction of the flow with an angle of inclination $\varphi$. The definition of the rest of the parameters is seen at the nomenclature section. In view of the aforementioned considerations, we present the following governing models:

$$
\begin{gathered}
\rho \frac{\partial w^{*}}{\partial t^{*}}=-\frac{\partial P^{*}}{\partial x^{*}}+\mu \frac{\partial^{2} w^{*}}{\partial y^{* 2}}+\rho g \beta_{C}\left(C^{*}-C_{\infty}\right) \cos \varphi-\frac{\mu}{k^{*}} w^{*}-\sigma B_{0}^{2} w^{*} \\
\frac{\partial C^{*}}{\partial t^{*}}=D_{m} \frac{\partial^{2} C^{*}}{\partial y^{* 2}}
\end{gathered}
$$

The geometry of the irregular channel in dimensional form is:

$$
y^{*}=R_{0}-\frac{\delta^{*}}{2}\left(1+\cos 2 \frac{\pi x^{*}}{\lambda}\right)
$$

The corresponding boundary conditions are as:

$$
\left.\begin{array}{llll}
w^{*}=0 & C^{*}=C_{\infty} \text { at } & y^{*}=0 \\
w^{*}=0 & C^{*}=C_{w} \text { at } & y^{*}=R
\end{array}\right\}
$$

We introduce the following non-dimensional conditions as:

$$
\left.\begin{array}{l}
x=\frac{x^{*}}{L} ; y=\frac{y^{*}}{R_{0}} ; w=\frac{w^{*}}{w_{0}} ; t=\frac{t^{*} v}{R_{0}^{2}} ; P=\frac{P^{*} R_{0}^{2}}{L \mu w_{0}} ; D a=\frac{k^{*}}{R_{0}^{2}}, \delta^{*}=\frac{\delta}{R_{T}} \\
G c=\frac{g \beta_{C}\left(C_{w}-C_{\infty}\right) R_{0}^{2}}{v w_{0}} ; \phi=\frac{C^{*}-C_{\infty}}{C_{w}-C_{\infty}} ; M^{2}=\frac{\sigma B_{0}^{2} R_{0}^{2}}{\mu} ; S c=\frac{v}{D_{m}}
\end{array}\right\}
$$

Using the dimensionless quantities to simplify equation (1)-(3), we have the following:

$$
\begin{gathered}
\frac{\partial w}{\partial t}=-\frac{\partial P}{\partial x}+\frac{\partial^{2} w}{\partial y^{2}}+\cos \varphi G c \phi-\frac{1}{D a} w-M^{2} w \\
\frac{\partial \phi}{\partial t}=\frac{1}{S c} \frac{\partial^{2} \phi}{\partial y^{2}}
\end{gathered}
$$

The geometry of the irregular channel in dimensionless form is:

$$
y=1-\frac{\delta}{2 R_{T}}(1+\cos 2 \pi x)
$$

The corresponding boundary conditions are as:

$$
\left.\begin{array}{llll}
w=0 & \phi=0 & \text { at } & y=0 \\
w=0 & \phi=1 & \text { at } & y=h
\end{array}\right\}
$$

where: $x=\frac{1}{L}\left(d_{0}+\frac{\lambda}{2}\right)$

\section{Analytical Solution}

Since the flow of blood through an arterial channel is as a result of the pumping action of the heart, we consider the pressure gradient, velocity and concentration profiles as a 
solution in the form:

$$
-\frac{\partial p}{\partial x}=P_{o} e^{i \omega t}
$$

So that, the velocity and temperature profile are represented as:

$$
\begin{aligned}
& w(y, t)=w_{0}(y) e^{i \omega t} \\
& \phi(y, t)=\phi_{o}(y) e^{i \omega t}
\end{aligned}
$$

Substituting equation (10) - (12) into equation (6) and (7), and by using the transformation $\chi=\frac{y}{h}$, equation (6) and (7) are simplified:

$$
\begin{gathered}
\frac{\partial^{2} w_{0}}{\partial \chi^{2}}-\beta_{1} w_{0}=P_{0}-\cos \varphi G c \phi_{0} \\
\frac{\partial^{2} \phi_{0}}{\partial \chi^{2}}-\beta_{2} \phi_{0}=0
\end{gathered}
$$

where $\beta_{1}=h\left(M^{2}+i \omega+\frac{1}{D a}\right)$ and $\beta_{2}=h i \omega S c$

The corresponding boundary conditions are as:

$$
\left.\begin{array}{llll}
w_{0}=0 & \phi_{0}=0 & \text { at } & \chi=0 \\
w_{0}=0 & \phi_{0}=e^{-i \omega t} & \text { at } & \chi=1
\end{array}\right\}
$$

Equation (14) has a solution of the form:

$$
\phi_{0}=A \sinh \left(\sqrt{\beta_{2}} \chi\right)+B \cosh \left(\sqrt{\beta_{2}} \chi\right)
$$

Using equation (15) to solve for the function coefficients in equation (16) as:

$$
\phi_{0}=\left(\frac{e^{-i \omega t}}{\sinh \left(\sqrt{\beta_{2}}\right)}\right) \sinh \left(\sqrt{\beta_{2}} \chi\right)
$$

Substitute (17) into (13) and solve for the velocity profile with the concentration effect:

$$
\frac{\partial^{2} w_{0}}{\partial \chi^{2}}-\beta_{1} w_{0}=P_{0}-\beta_{3} \sinh \left(\sqrt{\beta_{2}} y\right)
$$

where $\beta_{3}=\left(\frac{\cos \varphi G c e^{-i \omega t}}{\sinh \left(\sqrt{\beta_{2}}\right)}\right)$

Equation (18) has a homogenous solution in the form:

$$
w_{h}(\chi)=A_{1} \sinh \left(\sqrt{\beta_{1}} \chi\right)+B_{1} \cosh \left(\sqrt{\beta_{1}} \chi\right)
$$

We assume the particular solution to take the form:

$$
w_{p}=P+A_{2} \sinh \left(\sqrt{\beta_{2}} \chi\right)+B_{2} \cosh \left(\sqrt{\beta_{2}} \chi\right)
$$

Differentiating equation (20) to second order and substitute the result into (18), we have:

$$
w_{p}=-\frac{P_{0}}{\beta_{1}}+\left(\frac{\beta_{3}}{\beta_{1}-\beta_{2}}\right) \sinh \left(\sqrt{\beta_{2}} \chi\right)
$$

The general solution to the non homogenous differential equation in equation (18), we obtained:

$w_{0}(\chi)=A_{1} \sinh \left(\sqrt{\beta_{1}} \chi\right)+B_{1} \cosh \left(\sqrt{\beta_{1}} \chi\right)-\frac{P_{0}}{\beta_{1}}+\left(\frac{\beta_{3}}{\beta_{1}-\beta_{2}}\right) \sinh \left(\sqrt{\beta_{2}} \chi\right)$

We solve for the function coefficients of equation (22) using the boundary conditions in equation (15) as follows:

$$
\begin{aligned}
& A_{1}=-\left(\frac{P_{0}}{\beta_{1}}\right) \frac{\cosh \left(\sqrt{\beta_{1}}\right)}{\sinh \left(\sqrt{\beta_{1}}\right)}+\frac{P_{0}}{\beta_{1} \sinh \left(\sqrt{\beta_{1}}\right)}+\left(\frac{\beta_{3}}{\beta_{2}-\beta_{1}}\right) \frac{\sinh \left(\sqrt{\beta_{2}}\right)}{\sinh \left(\sqrt{\beta_{1}}\right)} \\
& B_{1}=\frac{P_{0}}{\beta_{1}}
\end{aligned}
$$

Substitute equation (17) and (22) into equation (11) and (12), we simply as:

$$
\begin{gathered}
\phi(\chi, t)=\left(\frac{\sinh \left(\sqrt{\beta_{2}} \chi\right)}{\sinh \left(\sqrt{\beta_{2}}\right)}\right) \\
w(\chi, t)=\left(A_{1} \sinh \left(\sqrt{\beta_{1}} \chi\right)+B_{1} \cosh \left(\sqrt{\beta_{1}} \chi\right)-\frac{P_{0}}{\beta_{1}}+\left(\frac{\beta_{3}}{\beta_{1}-\beta_{2}}\right) \sinh \left(\sqrt{\beta_{2}} \chi\right)\right) e^{i \omega t}
\end{gathered}
$$

The geometry of atherosclerosis is:

$$
h=1-\frac{\delta}{2 R_{T}}(1+\cos 2 \pi x)
$$

\section{Simulated Graphical Results}

In the previous sections we formulated and solved mathematical models for the lipid concentration effect on blood flow through an inclined channel with magnetic field, using regular perturbation method. This section deals with simulation of the analytical solution in equation (24), (25) and (26) by varying the biophysical parameters values such as

$D a=5, M=3, S c=0.22, \delta=3, f=1.2, \lambda=0.3, G c=10, \varphi=15$ , these parameters values are varied within a specific range in order to actually see the effect of them on blood velocity, as well as lipid concentration profile, with the treatment parameter $R_{T}$ fixed as a little control. The graphical results are presented in Figure 1 - Figure 28 for velocity profile $w(\chi, t)$, concentration profile $\phi(\chi, t)$, volumetric flow rate $Q$, shear stress $\tau_{w}$, the rate of mass transfer $S h$. 


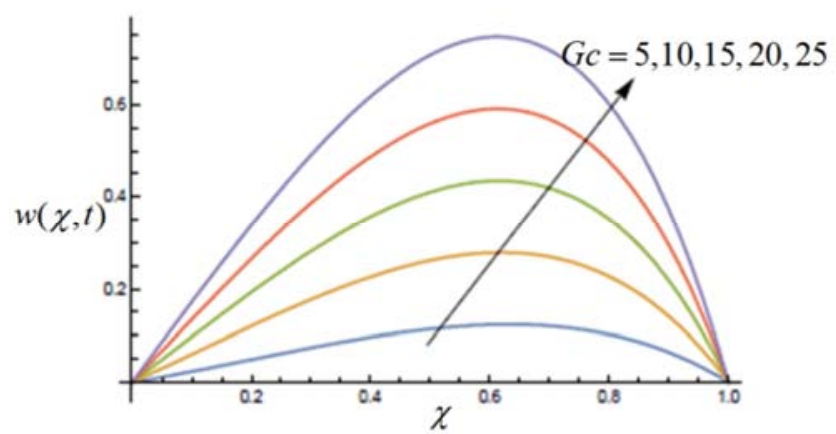

Figure 1. Influence of $G c$ on velocity profile.

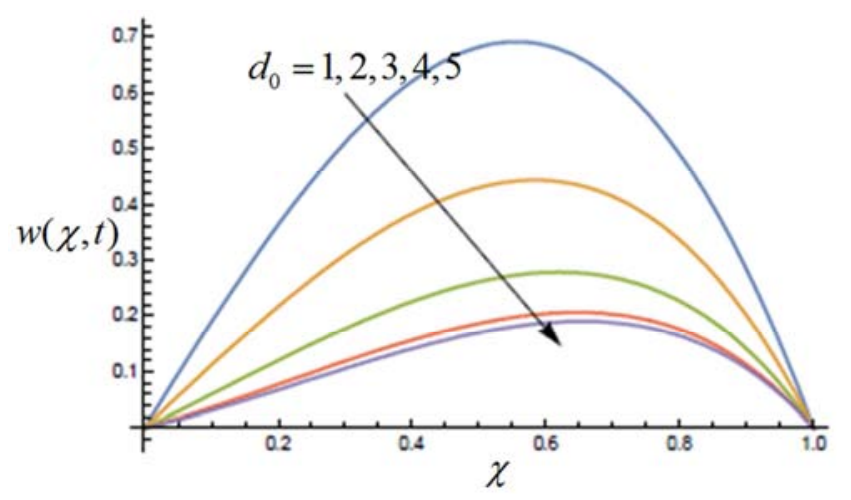

Figure 2. Influence of $d_{0}$ on velocity profile.

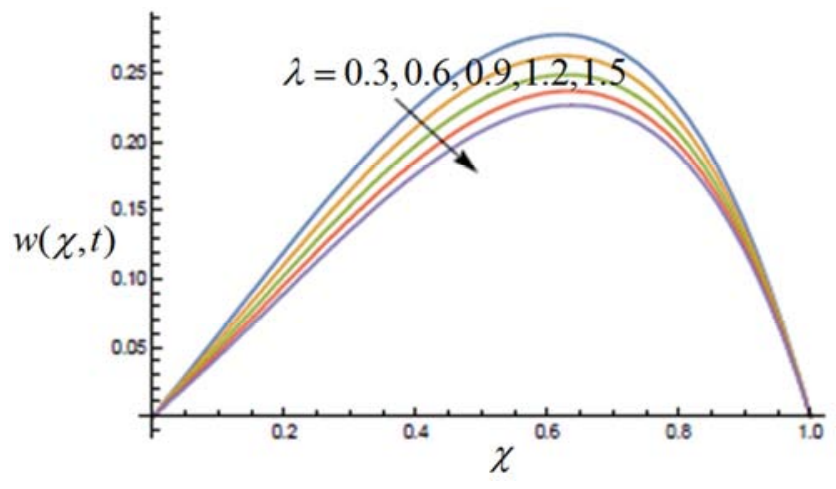

Figure 3. Influence of $\lambda$ on velocity profile.

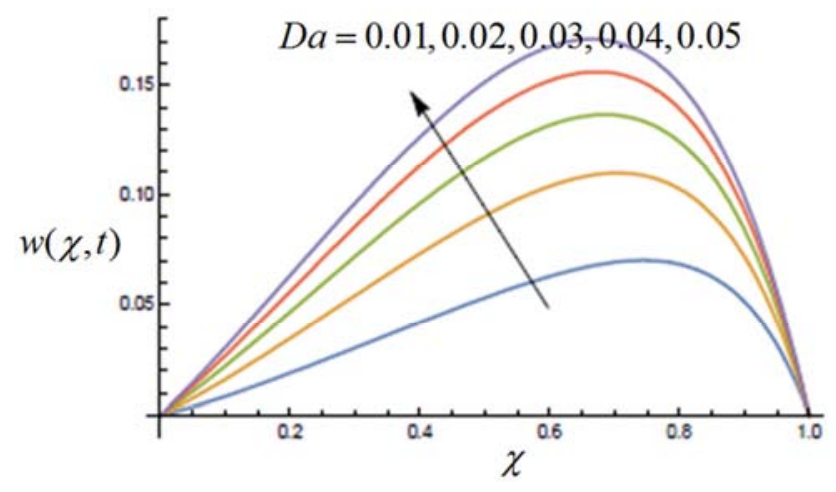

Figure 4. Influence of Da on velocity profile.

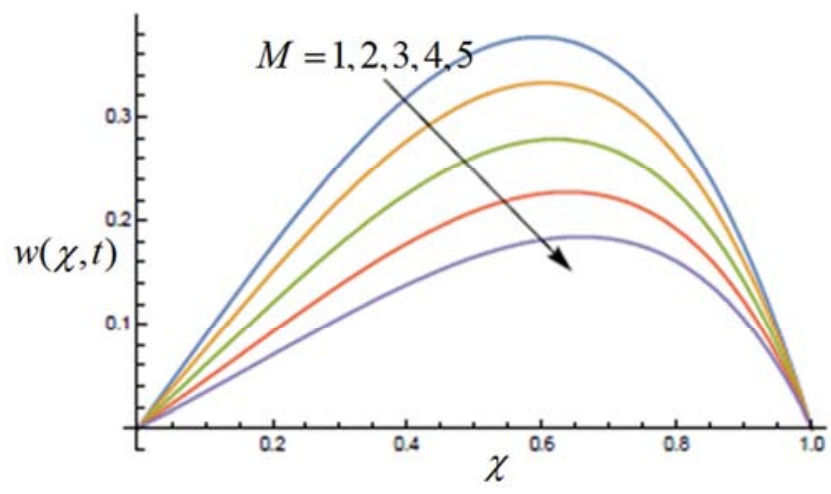

Figure 5. Influence of $M$ on velocity profile.

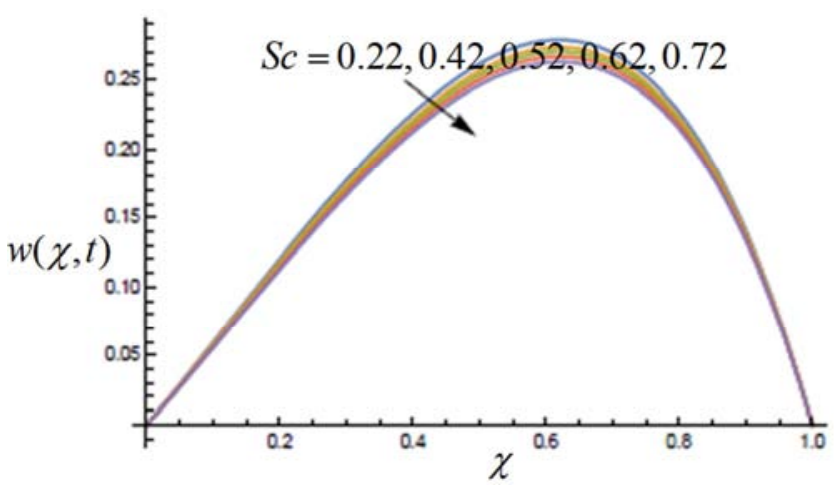

Figure 6. Influence of Sc on velocity profile.

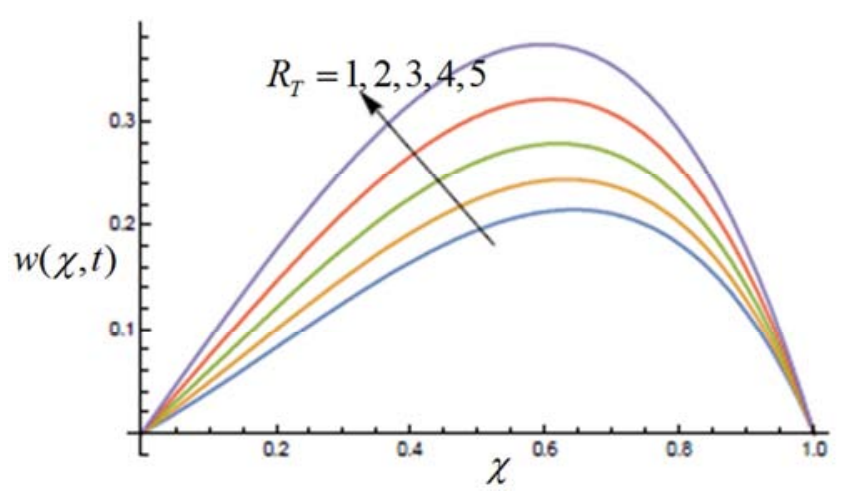

Figure 7. Influence of $R_{T}$ on velocity profile.

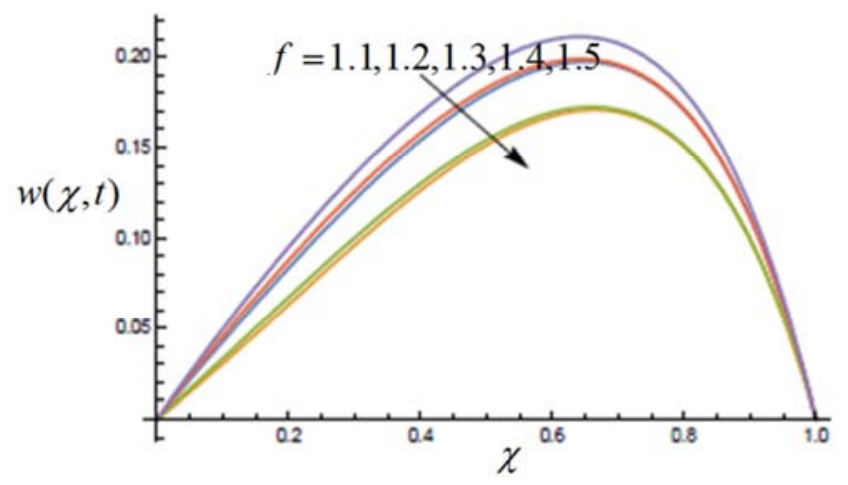

Figure 8. Influence of $f$ on velocity profile. 


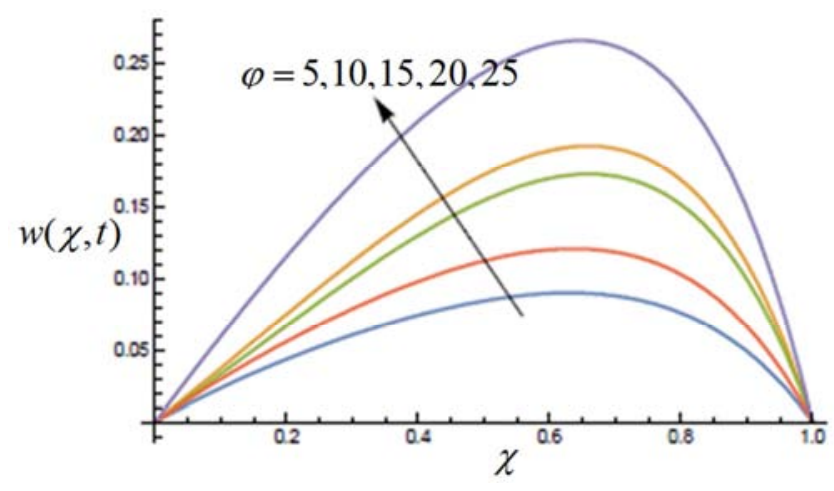

Figure 9. Influence of $\varphi$ on velocity profile.

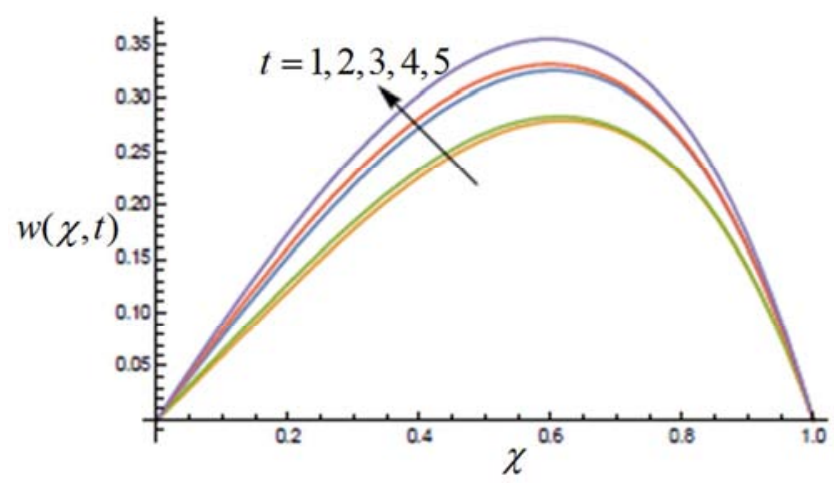

Figure 10. Influence of $t$ on velocity profile.

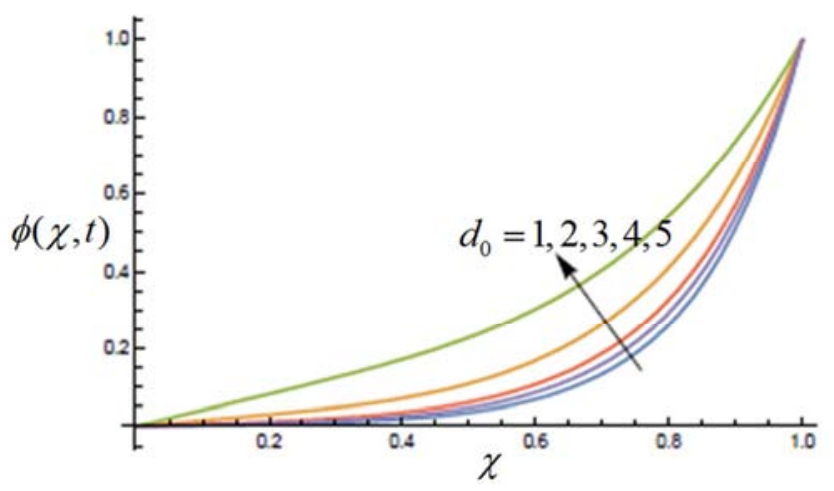

Figure 11. Influence of $d_{0}$ on concentration profile.

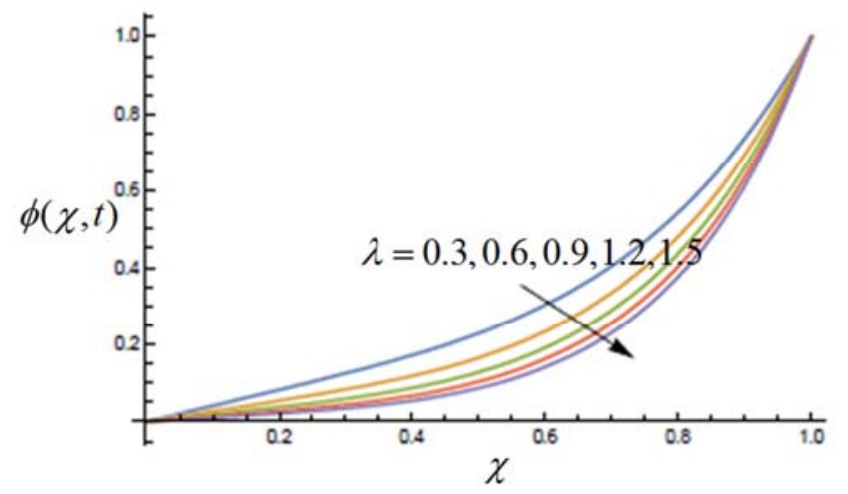

Figure 12. Influence of $\lambda$ on concentration profile.

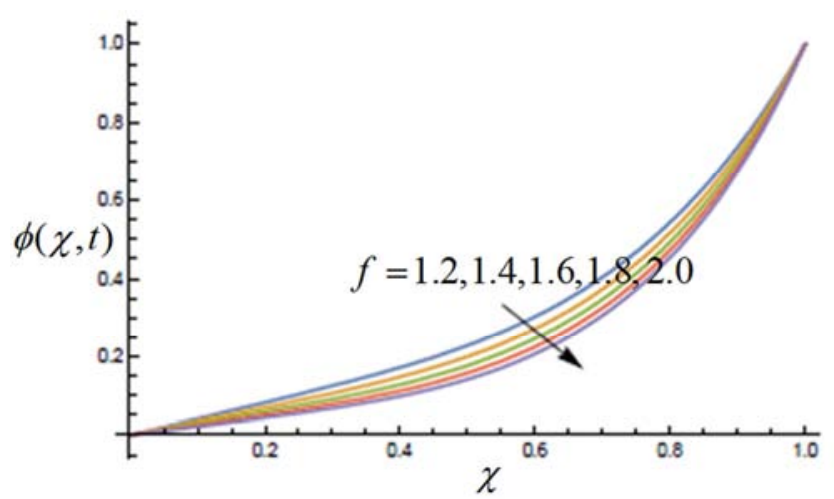

Figure 13. Influence of $f$ on concentration profile.

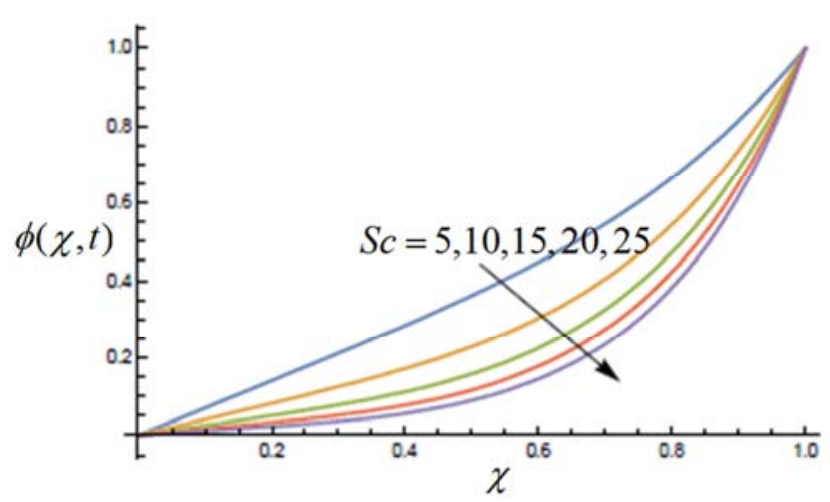

Figure 14. Influence of Sc on concentration profile.

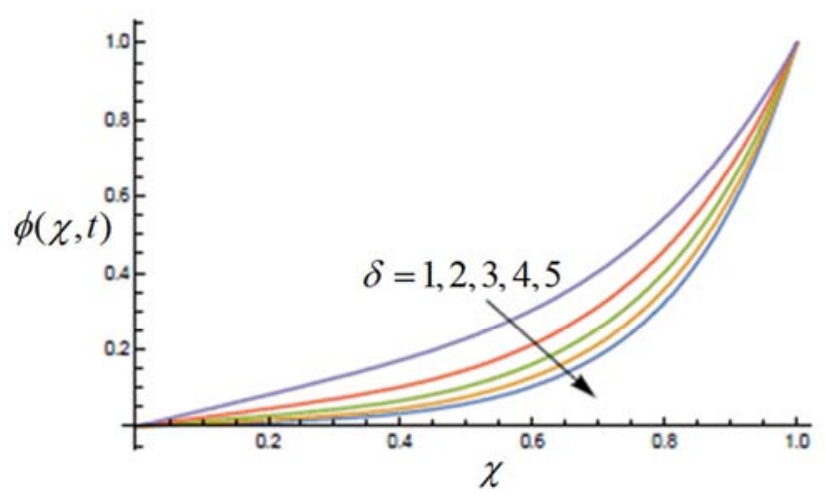

Figure 15. Influence of $\delta$ on concentration profile.

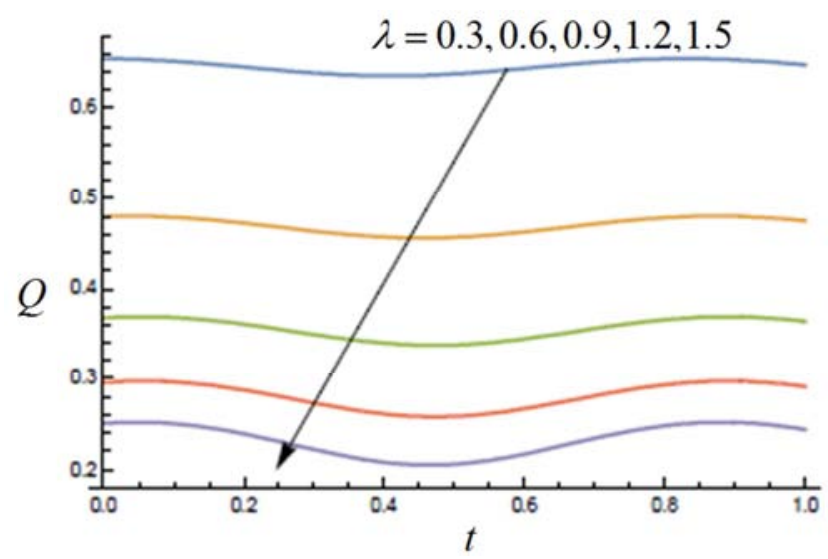

Figure 16. Influence of $\lambda$ on volumetric flow rate. 


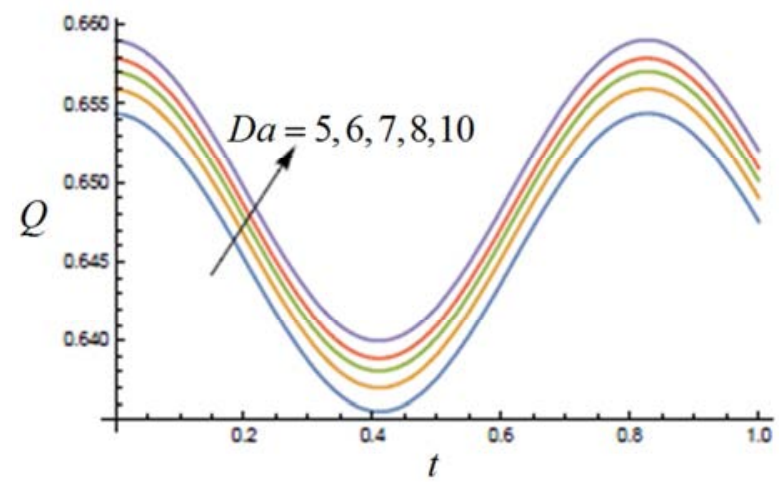

Figure 17. Influence of Da on volumetric flow rate.

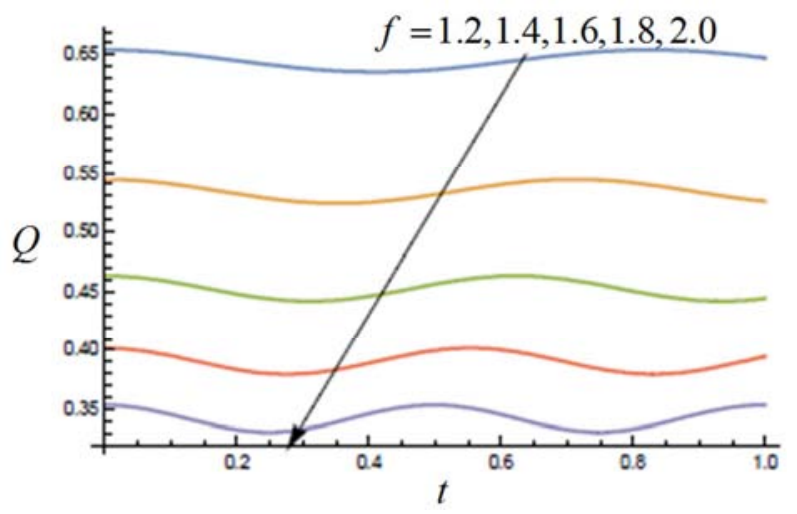

Figure 18. Influence of $f$ on volumetric flow rate.

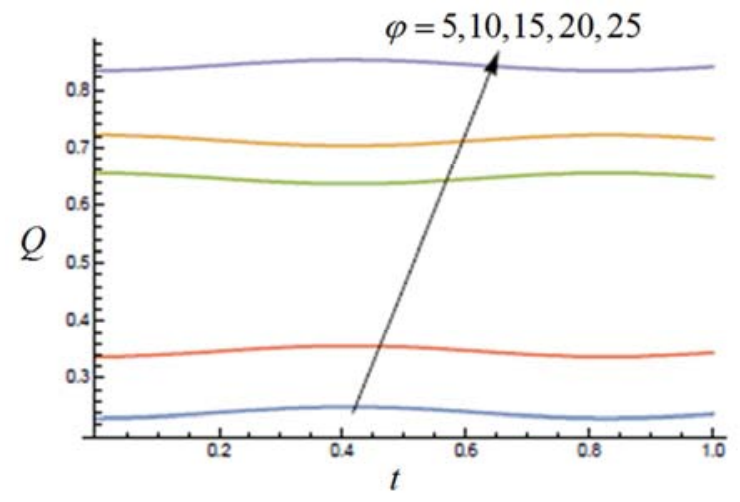

Figure 19. Influence of $\varphi$ on volumetric flow rate.

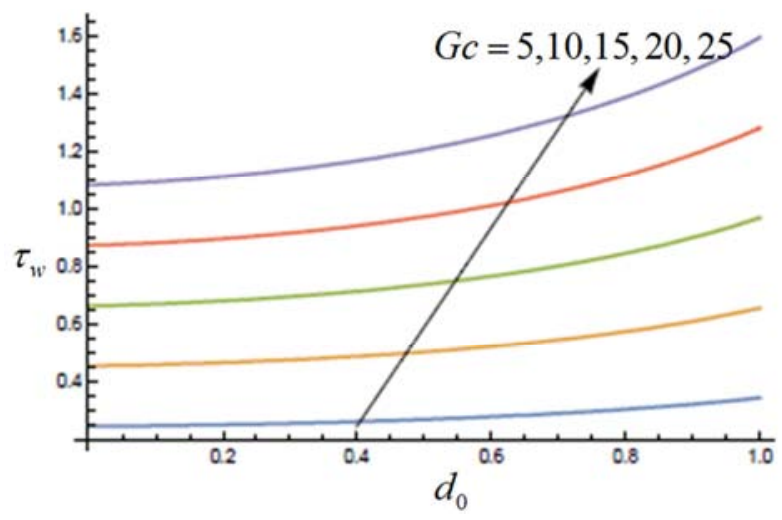

Figure 20. Influence of Gc on wall shear stress.

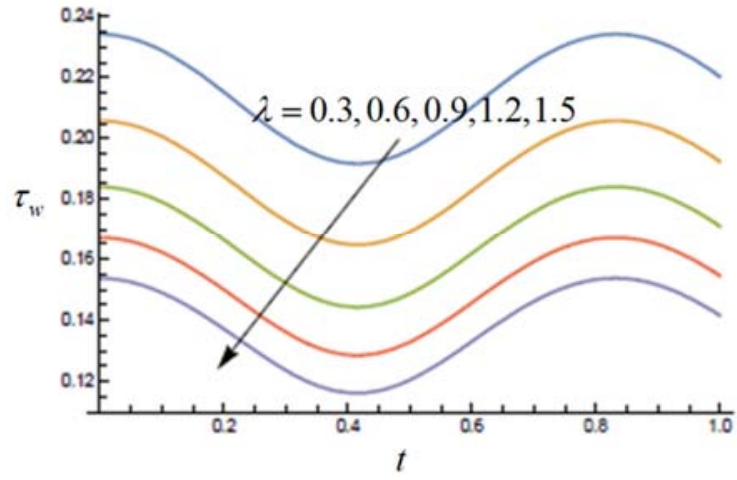

Figure 21. Influence of $\lambda$ on wall shear stress.

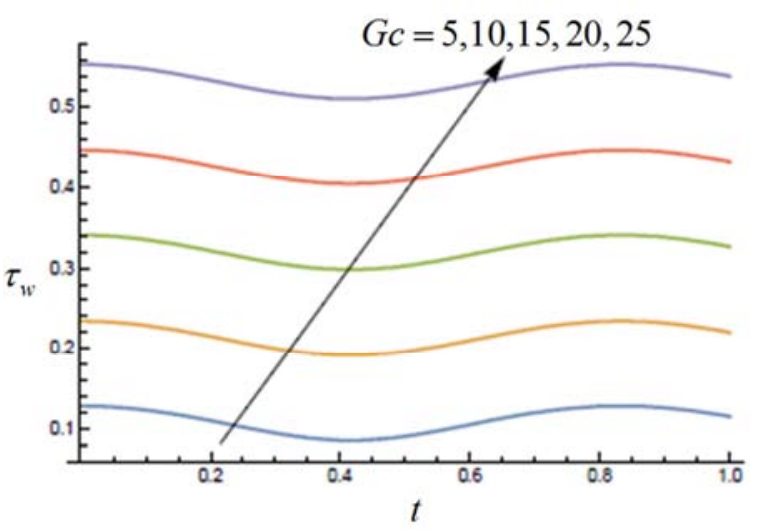

Figure 22. Influence of Gc on wall shear stress.

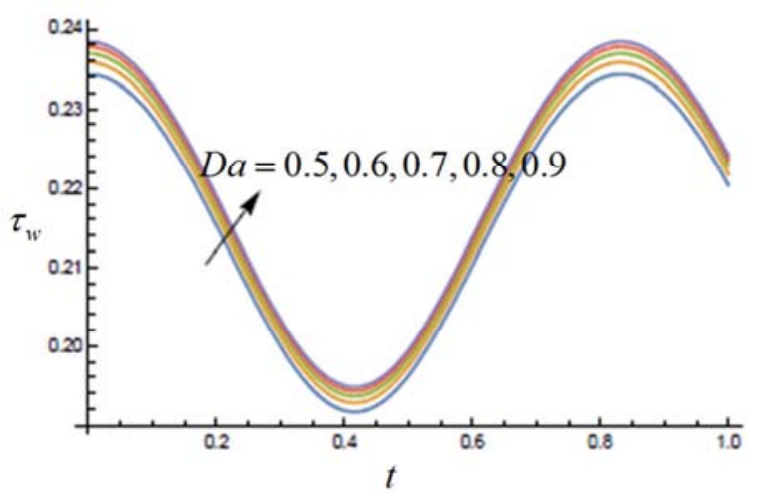

Figure 23. Influence of Da on wall shear stress.

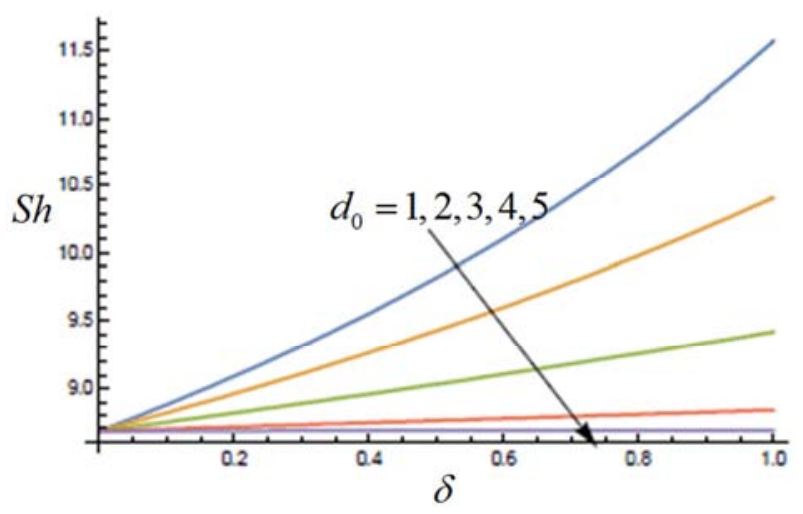

Figure 24. Influence of $d_{0}$ on Sherwood number. 


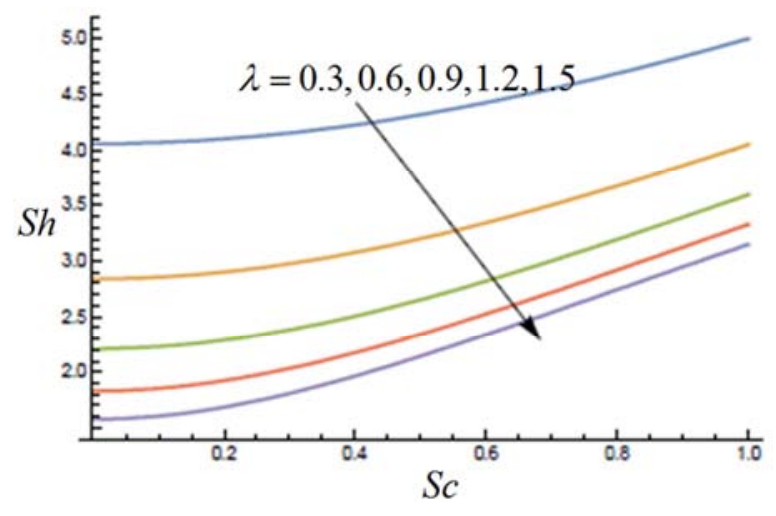

Figure 25. Influence of $\lambda$ on Sherwood number.

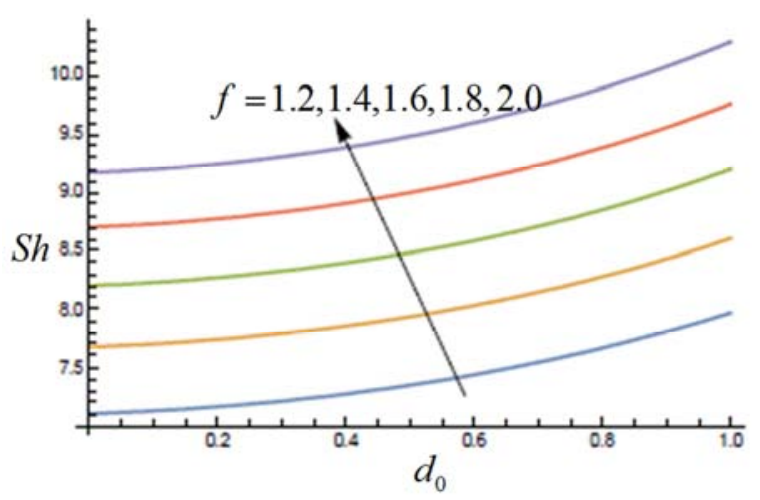

Figure 26. Influence of $f$ on Sherwood number.

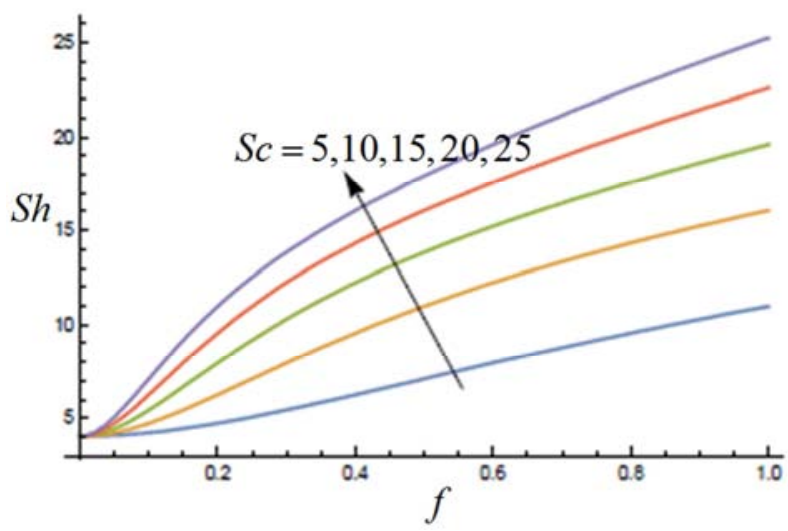

Figure 27. Influence of Sc on Sherwood number.

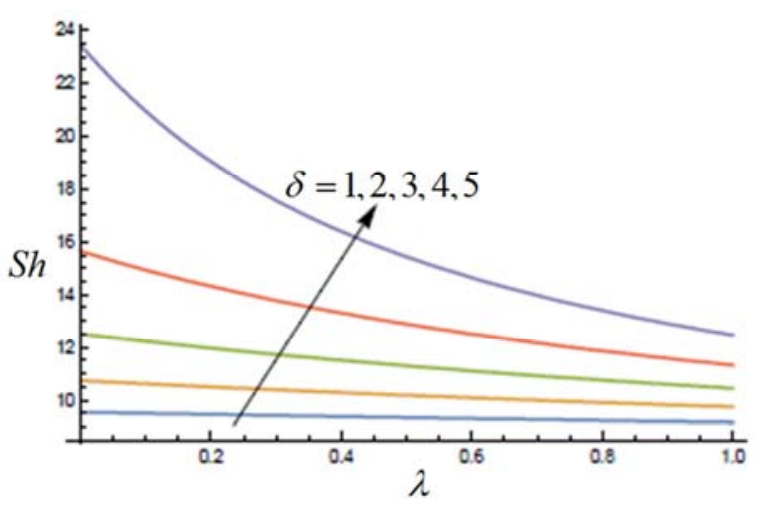

Figure 28. Influence of $\delta$ on Sherwood number.

\section{Discussion of the Graphical Results}

This section deals with the discussion of the graphically results on the basis of the variations of pertinent parameters, and their significant on blood velocity, concentration of lipid profile, volumetric flow rate, shear stress at the wall and the rate of mass transfers. In Figure 1, it is seen that increase in the solutal Grashoff number, increases the blood velocity in the arterial channel.

The importance of the onset of stenosis is illustrated in Figure 2; this figure depicts that increase in onset parameter, resulted to a decrease in the blood velocity. The onset is the point where the stenosis is first seen and thought of, in this investigation, its increase means an increase in the lipid concentration and density which decelerates the peak of flow.

The length of stenosis in the region of concern is noticed in Figure 3, it shows that the increase in the length of stenosis triggers a decrease in blood velocity. This result is so important and could be of help in arterial blood flow during surgical procedure. Porosity of any medium helps in any flow profile, as it is seen in Figure 4 . The figure show that increase in Darcy number resulted to a corresponding increasing in blood speed in the channel, and this is true and it agrees with existing biophysical laws. It is seen in Figure 5 that the increase in magnetic field intensity decelerates the blood velocity. This is of the fact that if magnetic field is applied to a moving electrically conducting fluid, it induces a force called the Lorentz force, which opposes or impede the flow. The relationship between the momentum diffusion and the mass diffusion is illustrated in Figure 6. This figure shows that increase in the Schmidt number resulted to a decrease in blood velocity because of the viscous diffusion rate is greater than the molecular diffusion rate. Figure 7 is a result for increasing values of the height of stenosis at certain level of porosity. This results show that increase in height of stenosis occasioned an increase the velocity profile because of the effectiveness of the treatment parameter in this cases. The pulse rate is related to both the systolic and diastolic blood pressures, and increase in pulse simply means increase in the systolic pressure under this investigation. This is an indication that the heart is over working due to high level of lipids in the system, as illustrated in Figure 8. The angle of inclination is very important in blood circulation during and after surgical procedure. That is so because it tells how a patient should be position while undergoing surgical procedure so that blood circulation would be under check, as displayed in Figure 9, in this figure, it is seen that increase in the angle of inclination increases the peak of the blood velocity.

Figure 10 depicts that an increase in the onset of stenosis increases the lipid concentration holding every other parameters values. This is so because the treatment has been able to take care of the lipid in circulation before it could clog somewhere in the lumen.

The length of stenosis and pulse rate parameter effects is 
seen in Figure 11 - Figure 12. In figure 11 we observed that the length parameters increase tend to decrease the concentration of the lipid in the channel. From our investigation, it is seen in Figure 13 - Figure 15 that concentration profiles decreases by increasing the values of the Schmidt number and the height of stenosis. It is seen also that increase in length of stenosis generates an increase in flow rate over time in Figure 16. Figure 17 illustrates the effect of Darcy number on blood flow rate; it shows that increase in the parameters increases the volumetric flow rate. The effect of pulse rate and angle of inclination is seen in Figure 18 - Figure 19. In Figure 20 - Figure 23, it is observed that increase in solutal Grashoff $G c$ and Darcy number $D a$ increases the shear stress but the stress decreases by increasing the length of stenosis.

Figure 24 - Figure 28 depicts that a decrease in rate of mass transfer with the increase in onset and length of stenosis parameters while the the rate increase with the increasing values of the pulse rate, Schmidt number and height of stenosis.

\section{Conclusion}

The lipid concentration effect on blood flow through an inclined arterial channel with focus on the length of stenosis, height of stenosis, pulse rate, angle of inclination, Darcy number, Solutal Grashoff number, and how these parameters affects lipid concentration profile and and blood flow profile with the help of the treatment parameter and external magnetic field. The governing equations with the considered boundary conditions were solved using perturbation technique and are simulated using Mathematica 10.

The study foundthe following:

a) The axial velocity profile increase is influenced by the treatment parameter, the solutalGrashoff number, the Darcy number, the angle of inclination and the time parameters. While the blood flow velocity decelerates with the increasing values of the onset of stenosis, the length of stenosis, magnetic field intensity parameters, Schmidt number and the pulse rate parameter.

b) In our investigation, we found that the onset of stenosis increase the lipid concentration level, while the length of stenosis, the pulse rate, the Schmidt number and the height of stenosis all decrease the lipid concentration level.

c) We found that the volumetric flow rate increases with the increasing values of the Darcy number and the angle of inclination while the flow rate decreases by increasing the values of the length and pulse rate parameters respectively.

d) The shear stress increases with the increasing values of the solutal Grashoff number and the Darcy parameter, while the stress decreases with length of stenosis increase.

e) The rate of lipid transfer increases with increase in pulse rate parameter, Schmidt number and the height of stenosis while the Sherwood number decreases with increasing values of the onset of stenosis and the length of stenosis.

\section{Nomenclature}

$\begin{array}{ll}y^{*} & \text { Dimensional coordinate variable } \\ y & \text { Dimensionless coordinate variable } \\ \chi & \text { Transformed coordinate variable } \\ x^{*} & \begin{array}{l}\text { Dimensional coordinate variable in the } \\ \text { horizontal direction }\end{array} \\ x & \text { Dimensionless coordinate variable in the } \\ R_{0} & \text { horizontal direction } \\ R(x) & \text { Radius of normal arterial segment } \\ \frac{\partial P}{\partial x} & \text { Radius of abnormal arterial segment } \\ \lambda & \text { Pressure gradient in }{ }^{x} \text { direction } \\ w^{*} & \text { Length of arterial segment } \\ w & \text { Dimensional blood velocity profile } \\ G c & \text { Dimensionless blood velocity profile } \\ \delta & \text { Dimensionless blood velocity profile } \\ R_{T} & \text { Dimensional maximum height of stenosis } \\ h & \text { Treatment parameter } \\ d_{0} & \text { Dimensionless atherosclerotic geometry } \\ t & \text { Onset of Stenosis } \\ \mu & \text { Time parameter } \\ \varphi & \text { Blood dynamic viscosity (0.0045Pa. s) } \\ \rho & \text { Angle of inclination under consideration } \\ f & \left.\text { Blood density (1050kg/cm }{ }^{3}\right) \\ \omega=2 \pi f & \text { Pulse rate } \\ & \text { Oscillatory parameter } \\ & \end{array}$

\section{Acknowledgements}

The authors would like to thank the reviewers for their motivating comments and useful suggestions in making this research paper successful.

\section{References}

[1] Bunonyo, K. W., Israel-Cookey, C., \& Amos, E. (2018). Modeling of Blood Flow through Stenosed Artery with Heat in the Presence of Magnetic Field. Asian Research Journal of Mathematics, 1-14.

[2] Shanthi, M., Pekka, P. and Norrving, B. (2011) Global Atlas on Cardiovascular DiseasesPrevention and Control. World Health Organization in collaboration with world Heart Federation and World Stroke Organisation, 3-18.

[3] Li, J. and Huang, H. (2010) Effect of Magnetic Field on Blood Flow and Heat Transfer through a Stenosed Artery. Proceedings of $3 \mathrm{rd}$ International Conference on Biomedical Engineering and Informatics, Yantai, 16-18 October 2010, 028-2032. https://doi.org/10.1109/BMEI.2010.5639654. 
[4] Alshare, A. Tashtoush, B. and Elkhali, H. H. (2013) Computational Modelling of Non-Newtonina Blood Flow through Stenosed Arteries in the Presence of Magnetic Field. Journal of Biochemical Engineering, 135, 5-15.

[5] Habibi, M. R. and Ghasemi, M. (2011) Numerical Study of Magnetic Nanoparticles Concentration in Biofluid (Blood) under Influence of High Gradient Magnetic Field. Journal of Magnetism and Magnetic Materials, 321, 32-38. https://doi.org/10.1016/j.jmmm.2010.08.023.

[6] Mekheimer, K. S., Haroun, M. H. and Elkot, M. A (2012) Influence of Heat and Chemical Reactions on Blood Flow through an Isotropically Tapered Elastic Arterieswith Overlapping Stenosis. Applied Mathematics, 6, 281-292.

[7] Sharma, P. R., Sazid, A. and Katiyar, V. K. (2011) Mathematical Modelling of Heat Transfer in Blood Flow through Stenosed Artery. Journal of Applied Sciences Research, 7, 68-78.

[8] Srinivas, S., Vijayalakshmi, A. and Redely, A. S. (2017) Flow and Heat Transfer of Gold Blood Nanofluid in a Porous Channel with Moving/Stationary Wall. Journal of Mechanics, 33, 395-404.

[9] Yadav, R. P., Harminder, S. and Bhoopal, S. (2008) Experimental Studies on Blood Flow in Stenosis Arteries in the Presence of Magnetic Field. Ultra Sciences, 20, 499-504.

[10] Tiari, S., Ahmadpour, M., Tafazzoli-Shadpour, M. and Sadeghi, M. R. (2011) An Experimental Study of Blood Flow in a Model of Coronary Artery with Single and Double Stenosis. Proceedings of the 18th Iranian Conference on Biomedical Engineering, Tehran, 14-16 December 2011, 3336.

[11] Aiman, A. and Bourhan, T. (2016) Simulation of MHD in Stenosed Arteries in Diabetic or Anaemic Model. Computational and Mathematical Methods in Medicine, 2016, Article ID: 8123930.

[12] Misra, J. C. and Shit, G. C. (2007) Role of Slip Velocity in Blood Flow through Stenosed Arteries: A Non-Newtonian Model. Journal of Mechanical in Medicine and Biology, 7, 337-353. https://doi.org/10.1142/S0219519407002303.

[13] Ponalgusamy, R. (2007) Blood Flow through an Artery with Stenosis. A TwoLayered Model, Different Shape of Stenosis and Slip Velocity at the Wall. Journal of Applied Sciences, 7, 1071-1077. https://doi.org/10.3923/jas.2007.1071.1077.

[14] Verma, N. K., Siddiqui, S. U., Gupta, R. S. and Mishra, S. (2011) Effect of Slip Velocity on Blood Flow through a Catheterized Artery. Applied Mathematics, 2, 764-770. https://doi.org/10.4236/am.2011.26102.
[15] Guar, M. and Gupta, M. K. (2014) Steady Slip Blood Flow through a Stenosed Porous Artery. Advanced in Applied Sciences Research, 5, 249-259.

[16] Srikanth, D. S., Ramana, R. S. and Jain, A. K. (2015) Unsteady Polar Fluid Model of Blood Flow through Tapered X-Shape Stenosed Artery. Effect of Catheter and Velocity Slip. Ain Shams Engineering Journal, 6, 10931104.https://doi.org/10.1016/j.asej.2015.01.003.

[17] Arun, K. M. (2016) Multiple Stenotic Effect of Blood Flow Characteristic in the Presence of Slip Velocity. American Journal of Applied Mathematics and Statistics, 4, 154-198.

[18] Geeta, A. and Siddique, S. U. (2016) Analysis of Unsteady Blood Flow through Stenosed Artery with Slip Effect. International Journal of Bio-Science and Bio-Technology, 8, $43-54$.

[19] Sanjeev, K. and Chandraahekhar, D. (2015) Hematocrit Effect of the Axisymmetric Blood Flow through an Artery with Stenosed Arteries. International Journal of Mathematics Trends and Technology, 4, 91-96.

[20] Verma, N. K. and Parihar, R. S. (2010) Mathematical Model of Blood Flow through a Tapered Artery with Mild Stenosed and Hematocrit. Journal of Applied Mathematics and Computer, 1, 30-46.

[21] Shit, G. C. and Screeparma, M. (2015) Pulsatile Flow of Blood and Heat Transfer with Variable Viscosity under Magnetic and Vibration Environment. Journal of Magnetism and Magnetic Materials, 388, 106-115. https://doi.org/10.1016/j.jmmm.2015.04.026.

[22] Singh, J. and Rathee, R. (2010) Analytical Solution of Two Dimensional Model of Blood Flow with Variable Viscosity through an Indented Artery Due to LDL Effect in the Presence of Magnetic Field. International Journal of Physical Sciences, $5,1851-1868$

[23] Chitra, M. and Karthikeya, D. (2017) Oscillatory Flow of Blood in Porous Vessel of a Stenosed Artery with Variable Viscosity under the Influence of Magnetic Field. International Journal of Innovative Research in Advanced Engineering, 4, $52-60$.

[24] Jagdish, S. and Rajbala, R. (2010) Analytical Solution of Two Dimensional Model of Blood Flow with Varible Viscosity through an Indented Artery due to LDL Effect in the Presence of Magnetic Field. International Journal of Physical Sciences, $5,1857-1868$.

[25] Bunonyo, K. W., \& Amos, E. (2020)."Investigation of the Treatment and Radiation Effects on Oscillatory Blood Flow through a Stenosed Artery."American Journal of Engineering Research (AJER), vol. 9 (04), pp. 253-259. 